\title{
POSITIONAL COINCIDENCE BETWEEN WATER MASERS AND A PLASMA TORUS IN NGC 1052
}

\author{
Satoko Sawada-Satoh ${ }^{1}$, Seiji Kameno ${ }^{2}$, Katsunori M. Shibata ${ }^{2}$, and Makoto Inoue ${ }^{2}$ \\ ${ }^{1}$ Academia Sinica Institute of Astronomy and Astrophysics, P.O. Box 23-141, Taipei 106, TAIWAN \\ E-mail: satoko@asiaa.sinica.edu.tw \\ ${ }^{2}$ National Astronomical Observatory of Japan, Mitaka, Tokyo 181-8588, JAPAN \\ (Received February 1, 2005; Accepted March 15, 2005)
}

\begin{abstract}
We present results of the VLBA observation toward the radio continuum and water maser emissions in a nearby LINER galaxy NGC 1052. The jet structure observed in 2000 is similar to that in 1998, and the two jet structures in 1998 and 2000 support the sub-luminal motion with apparent velocity of $0.26 \mathrm{c}$. Distribution of water maser spots are located $\sim 0.05 \mathrm{pc}$ shifted to southwest from the component which is supported to be the nucleus, and no rapid positional change of the water maser gas with respect to the central engine is seen from 1995 to 2000 . The maser gas is positionally coincident with a plasma torus, and the position of the maser gas relative to the nucleus is stable from 1995 to 2000 . The maser gas in NGC 1052 could be explained to be associated with the nuclear circumnuclear torus or disk like the situation found in the nucleus of NGC 4258.
\end{abstract}

Key words : galaxies: active — galaxies: individual (NGC 1052, PKS B0238-084, J0241-0815) — galaxies: nuclei - masers - radio lines: galaxies

\section{INTRODUCTION}

NGC 1052, a nearby radio galaxy, has double-sided jets at radio (Jones et al. 1984, Wrobel 1984) and optical and X-ray (Kadler et al. 2004a) wavelengths. Parsec-scale jets were imaged with VLBI at several frequencies (Claussen et al. 1998, Kellermann et al. 1998, Kameno et al. 2001, Kameno et al. 2003, Vermeulen et al. 2003, Kadler et al. 2004b), which elongated in P.A. $\sim 65^{\circ}$. Proper motion between the two jets and structure evolution were seen in the galaxy (Kellermann et al. 1998). VLBA monitor observations showed the proper motion between the two jets with an apparent velocity of $0.26 \pm 0.04 \mathrm{c}$ from 1995 to 2001 (Vermeulen et al. 2003). Using the ratio of brightness temperatures in the eastern and western jets, Kameno et al. (2001) suggested that the jet axis inclines by $\sim 50^{\circ}$ from the line of sight. Vermeulen et al. et al. (2003) claimed that the jet axis should be close to the sky plane with the lower limit of the inclination of $57^{\circ}$. Kadler et al. (2004b) derived the maximum allowed angle to the line of sight is $72^{\circ}$.

A gap between the east and west jets, where the nucleus is supposed to be, had been recognized till 1999 (Claussen et al. 1998, Kellermann et al. 1998, Vermeulen et al. 2003, Kadler et al. 2004b). In 2000, however, the nuclear component appeared and was identified between the east and west jets (Kameno et al. 2001). Kameno et al. (2001) found a central condensation of the plasma which covers about $0.1 \mathrm{pc}$

Proceedings of the 6th East Asian Meeting of Astronomy, held at Seoul National University, Korea, from October 18-22, 2004. and $0.7 \mathrm{pc}$ of the approaching and receding jets, respectively. From the results, Kameno et al. (2001) proposed a parsec-scale circumnuclear torus model in NGC 1052. Alignment of multi-frequency images performed by Kadler et al. (2004b) revealed that component B derived by Kameno et al. (2001) is likely the most inner component of western jet in 1998 .

NGC 1052 emits a water megamaser, which is redshifted with respect to the systemic velocity of the galaxy (1491 $\mathrm{km} \mathrm{s}^{-1}$; de Vaucouleurs et al. 1991). The spectral profile shows a broad velocity widths of $\sim 100 \mathrm{~km} \mathrm{~s}^{-1}$ (FWHM) (Braatz et al. 1994). Monitoring observations of water maser emission over eight years find variability of the spectral profile (Braatz et al. 1996, Braatz et al. 1997, Braatz et al. 2003). In 2003, the new narrow maser feature at $V_{\text {LSR }}$ $=1787 \mathrm{~km} \mathrm{~s}^{-1}$ are detected with the NRO $45-\mathrm{m}$ telescope (Kameno et al. 2005).

Past VLBI observations of water maser emissions in November 1995 showed that the water maser gas with velocity range of $1585-1685 \mathrm{~km} \mathrm{~s}^{-1}$ consists of two clusters (Claussen et al. 1998). The clusters distributed along the western jet and $0.05-0.1 \mathrm{pc}$ shifted to the west from the gap, where the most inner component of western jet, or component B.

Claussen et al. (1998) explained that the maser gas is excited by shocks into dense molecular clump which lie in or around the radio jet, or amplification of the radio continuum emission of the jet by foreground molecular clouds. The explanation indicates that the maser gas in NGC 1052 is not associated with a Keplerian disk found in NGC 4258 as described by Miyoshi et al. (1995). A similar explanation of maser gas with- 


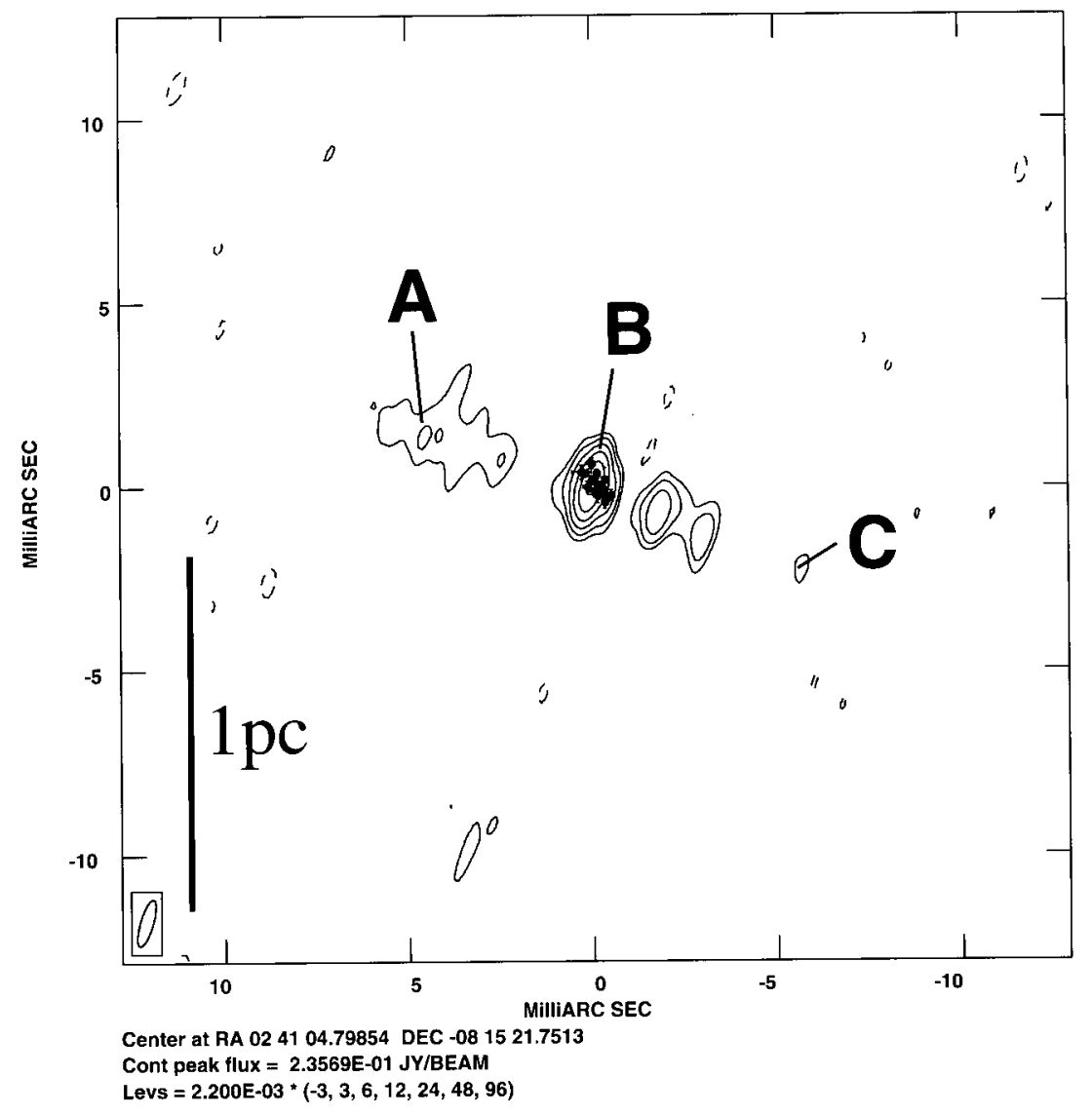

Fig. 1. - Distribution of the water maser spots (full circle) and the continuum components (contour) at $22 \mathrm{GHz}$ in NGC 1052. The labels A, B and C are the same nomenclature by Kameno et al. (2001). Synthesized beam size, shown in lower left corner is $1.0 \times 0.48$ mas at a position angle of $-9.4^{\circ}$. Contour start at $\pm 3 \sigma$ levels, increasing by factors of 2 , where $\sigma=2.2 \mathrm{mJy}^{\text {beam }^{-1}}$.

out a disk in AGN was made for Mrk 348, whose maser arises from a region less than $0.25 \mathrm{pc}$ in extent along the receding jet (Peck et al. 2003).

In order to confirm the positional relation between the maser gas and the plasma circumnuclear torus, we observed the continuum and maser emissions in the nucleus of NGC 1052 with VLBA. We adopt $z=$ 0.0049 (Knapp et al. 1978) corresponds to the distance $D=20 \mathrm{Mpc}$ to NGC 1052 assuming $H_{0}=$ $75 \mathrm{~km} \mathrm{~s}^{-1} \mathrm{Mpc}^{-1}$ and $q_{0}=0.5$, hence 1 mas corresponds to $0.095 \mathrm{pc}$.

\section{OBSERVATIONS AND CALIBRATIONS}

Our VLBA observation toward NGC 1052 was carried out at $22 \mathrm{GHz}$ in July 2000 . We used four IF channels with a bandwidth of $8 \mathrm{MHz}$ and allocated the LSR velocity of $1000,1200,1650$ and $1750 \mathrm{~km} \mathrm{~s}^{-1}$ for each center of IF channel. The IF channels of 1650 and $1750 \mathrm{~km} \mathrm{~s}^{-1}$ cover the velocity range from 1560 to 1840 $\mathrm{km}^{-1}$, where the water maser emissions have been de- tected in. The line-free continuum emissions are taken with the IF channels of 1000 and $1200 \mathrm{~km} \mathrm{~s}^{-1}$. The data were recorded with a bit rate of $128 \mathrm{Mbps}$ at 2-bit sampling. 3C454.3 and 3C84 were used as calibrators during the observation. The correlation process was done using the NRAO VLBA correlator.

Data reduction including a priori amplitude calibration, data flagging, fringe fitting and imaging proceeded using the NRAO AIPS package. For continuum image, we averaged line-free continuum channels after correcting for the residual delay and delay rate. We applied the solutions of self calibration for the continuum channels to the water maser line channels. Using JMFIT task in AIPS, we measure the relative positions and sizes of water maser spots and continuum component. Imaging was performed with the natural weighting, resulting in the synthesized beam size of $1.0 \times 0.48$ mas at a position angle of $-9.4^{\circ}$. 


\section{RESULTS}

Fig. 1 shows the radio continuum image at $22 \mathrm{GHz}$ and the distribution of water maser spots in July 2000. We detected a bright nuclear component and doublesided jet components, and the continuum structure is similar to images of December 1998 at $15 \mathrm{GHz}$ by Kameno et al. (2001) and at $22 \mathrm{GHz}$ by Kadler et al. (2004b). Therefore, we use hereafter the same nomenclature for these components as those used in Kameno et al. (2001). Considering the proper motion of an apparent velocity of $0.26 c$ (Vermeulen et al. 2003), the component $\mathrm{A}$ and $\mathrm{C}$ should be shifted 5 mas east and 6 mas west, respectively. Positional differences of these components between Kameno et al. (2001) and ours are consistent with the proper motion. The doublepeaked component between $\mathrm{B}$ and $\mathrm{C}$ in our image can be a new jet component, which has been seen as an elongated component from $\mathrm{B}$ to $\mathrm{C}$ in image at $15 \mathrm{GHz}$ by Kameno et al. (2001) and image at $22 \mathrm{GHz}$ by Kadler et al. (2004b) already.

In our observation, maser emission within the velocity range of $1670-1760 \mathrm{~km} \mathrm{~s}^{-1}$ was detected significantly. No significant motion in the distribution of maser gas with respect to the component $\mathrm{B}$ was found since 1995, and the maser gas is located where freefree absorption opacity is large, or where the plasma torus covers foreground of the receding jet (Kameno et al. 2001). Detailed maser distribution is shown in Fig 2. The maser spots distributed within $0.1 \mathrm{pc}$ along the NE-SW direction, which agrees well with the alignment of two clusters Claussen et al. (1998) showed. Most of maser spots are inside the circle with radius of 0.47 mas, which is the maximum size of deconvolved component $\mathrm{B}$. B is located at $\Delta X, \Delta Y=(0.0 \pm 0.3 \mathrm{mas}$, $0.9 \pm 0.6$ mas, and its deconvolved size $<0.47$ mas.

\section{DISCUSSION}

Neufeld \& Maloney (1995) and Herrnstein et al. (1996) proposed an idea that a masering molecular disk in NGC 4258 includes several physical-condition layers inside. Kameno et al. (2005) applied their idea to circumnuclear torus model in NGC 1052 demonstrated by Kameno et al. (2001). Our results show the positional coincidence between water maser gas and a torus in NGC 1052, and strongly support the scenario described by Kameno et al. (2005). Fig. 2 shows a schematic torus structure of NGC 1052. A plasma layer is created on the inner surface of the torus, because the gas in the surface is directly exposed to the Xray radiation from the nucleus. The inner plasma layer causes free-free absorption. X-ray dissociation region $(\mathrm{XDR})($ Maloney 2002) lies next to the plasma layer inside the torus, heated to temperature (above $\sim 400 \mathrm{~K}$ ) for collisional pumping of masers by X-ray radiation. Excited molecules in XDR amplify the continuum seed emission from the jet component in back ground and emit maser emissions. Gas inside the torus is accret-

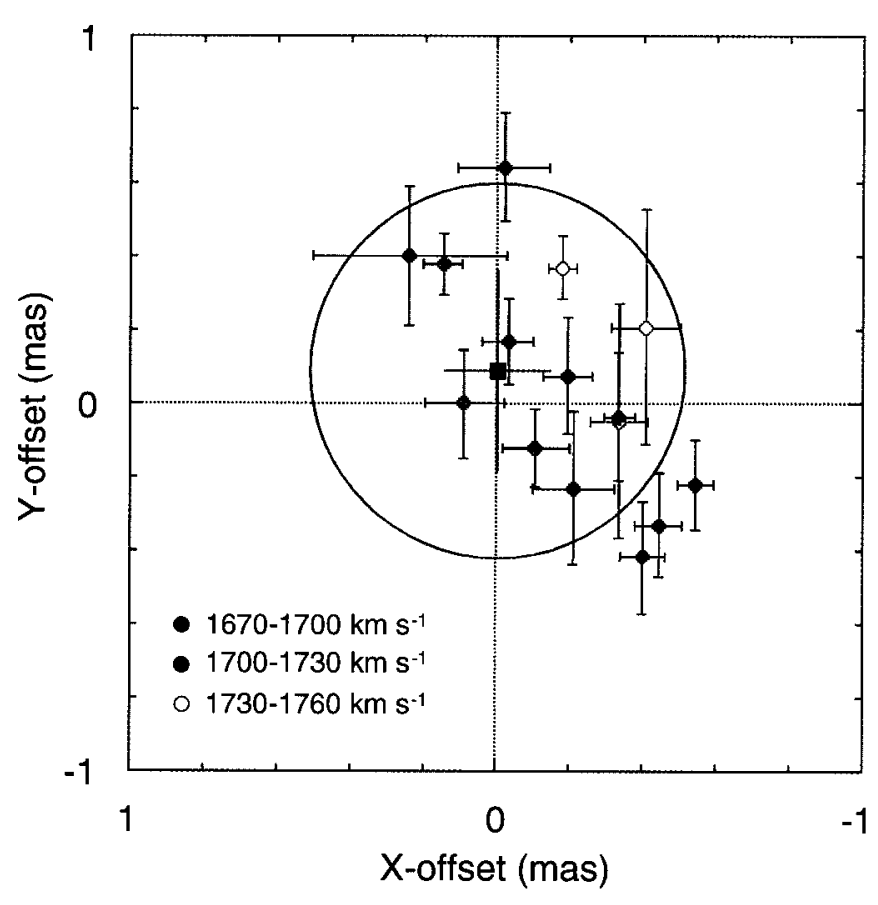

Fig. 2.- Detailed distribution of the water maser spots and the continuum component B in NGC 1052. Color full circles indicate the velocity shift of water maser emission and a black full square is the position of the component $\mathrm{B}$. Big open circle is the maximum size of deconvolved the component $B$ with the synthesized beam.

ing onto the black hole in the nucleus. This modified torus model explains not only why maser spots distribute only on the western jet where free-free absorption opacity is significant, but also why the maser emission is redshifted from the systemic velocity of the galaxy. When a new jet component ejects and runs behind the XDR, redshifted maser emissions can be brighter.

Another possible explanation is an excitation of maser gas by jet as interpreted the case of Mrk 348 (Peck et al. 2003). If the maser gas is moving with the most redshifted velocity $\left(\sim 400 \mathrm{~km} \mathrm{~s}^{-1}\right)$ from the systemic velocity of the galaxy, the maser gas should move $1.9 \times 10^{-3}$ pc east from November 1995 to July 2000 , because the jet axis is close to the sky plane. This motion is too small to detect for five-year multi-epoch observations. Further VLBI observations are necessary in order to detect the proper motion of water maser gas.

\section{REFERENCES}

Braatz, J. A., Wilson, A. S., \& Henkel, C., 1994, ApJ, 437, L99

Braatz, J. A., Wilson, A. S., \& Henkel, C., 1996, ApJS, 106, 51

Braatz, J. A., Wilson, A. S., \& Henkel, C., 1997, ApJS, 110, 321 


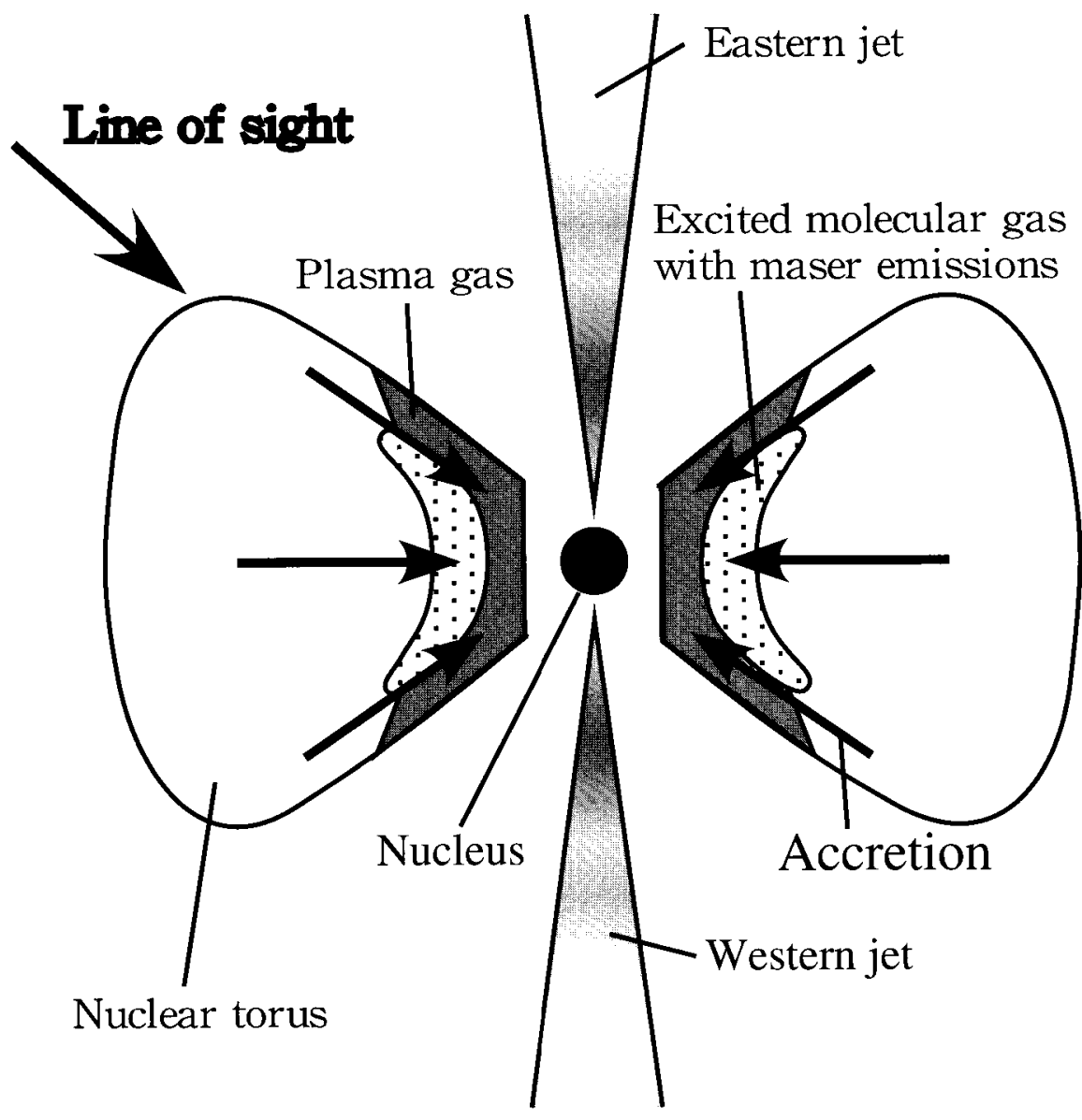

Fig. 3.- A possible environment in the circumnuclear torus and jets in NGC 1052. Angle of the jet axis from the line of sight is $50^{\circ}$. Inner surface of the torus is ionized by X-ray emission. X-ray dissociation region (XDR) is formed on the inner layer of the torus and amplify background continuum emission from the jet.

Braatz, J. A., Wilson, A. S., Henkel, C., Gough, R., \& Sinclair, M., 2003, ApJS, 146, 249

Claussen, M. J., Diamond, P. J., Braatz, J. A., Wilson, A. S., \& Henkel, C., 1998, ApJ, 500, L129

de Vaucouleurs, G., 1991, Third Reference Catalogue of bright galaxies

Herrnstein, J. R. Greenhill, L. J., \& Moran, J. M., 1996, ApJ, 468, L17

Jones, D. L., Wrobel, J. M., \& Shaffer, D. B., 1984, ApJ, 276,480

Kadler, M., Kerp, J., Ros, E., Falcke, H., Pogge, R. W., \& Zensus, J. A., 2004(a), A\&A, 420, 467

Kadler, M., Ros, E., Lobanov, A. P., Falcke, H., \& Zensus, J. A., 2004(b), A\&A, 426, 481

Kameno, S., Sawada-Satoh, S., Inoue, M., Shen, Z., \& Wajima, K., 2001, PASJ, 53, 169

Kameno, S., Inoue, M., Wajima, K., Sawada-Satoh, S., \& Shen, Z.-Q., 2003, Publ. of the Astron. Soc. of Australia, 20, 134
Kameno, S., Nakai, N., Sawada-Satoh, S., Sato, \& N., Haba, A., 2005 in press

Kellermann, K. I., Vermeulen, R. C., Zensus, J. A., \& Cohen, M. H., 1998, AJ, 115, 1295

Knapp, G. R., Faber, S. M., \& Gallagher, J. S., 1978, AJ, $174, \mathrm{~L} 123$

Maloney, P. R., 2002, Publications of the Astronomical Society of Australia, 19, 401

Neufeld, D. A. \& Malony, P. R., 1995, ApJ, 447, L17

Peck, A. B., Henkel, C., Ulvestad, J. S., Brunthaler, A., Falcke, H., Elitzur, M., Menten, K. M., \& Gallimore, J. F., 2003, ApJ, 590, 149

Vermeulen, R. C., Ros, E., Kellermann, K. I., Cohen, M. H., Zensus, J. A., \& van Langevelde. H. J., 2003, A\&A, 401,113

Wrobel, J. M., 1984, ApJ, 284, 531 